ISSN: 2602-8506

Vol. 3, N4., p.05-19, octubre - diciembre, 2019

\title{
La neuroeconomía y su contribución a la teoría del comportamiento del consumidor
}

The neuroeconomy and its contribution to the consumer behavior theory

Jacqueline Carolina Sánchez Lunavictoria. ${ }^{1}$, Carlos Augusto Delgado Rodriguez. ${ }^{2}$, Paulina Fernanda Bolaños Logroño. ${ }^{3}$ \& Jenny Isabel Chávez Rojas. ${ }^{4}$

\section{DOI: https://doi.org/10.33262/visionariodigital.v3i4.949}

\begin{abstract}
This document addresses in its theoretical context the contribution of neuroeconomics to the theory of consumer behavior, in its practical context the research presented has as its main objective to determine which are the most important factors that characterize the behavior of consumption of the inhabitants from the city of Riobamba, results that will serve as a consultation for future research or to refine strategies in the field of marketing
\end{abstract}

Keywords: Neuroeconomics, neuroscience, consumer behavior, neuromarketing

\section{Resumen}

El presente documento aborda en su contexto teórico el aporte de la neuroeconomía a la teoría del comportamiento del consumidor, en su contexto práctico la investigación que se presenta tiene como principal objetivo determinar cuáles son los factores más importantes que caracterizan el comportamiento de consumo de los habitantes de la ciudad de Riobamba, resultados que servirán de consulta para futuras investigaciones o para afinar estrategias en el campo de la mercadotecnia.

\footnotetext{
${ }^{1}$ Escuela Superior Politécnica de Chimborazo, Facultad de Administración de Empresas. Riobamba, Ecuador. carolina.sanchez@espoch.edu.ec

${ }^{2}$ Escuela Superior Politécnica de Chimborazo, Facultad de Administración de Empresas. Riobamba, Ecuador. carlos.delgado@espoch.edu.ec

${ }^{3}$ Escuela Superior Politécnica de Chimborazo, Facultad de Administración de Empresas. Riobamba, Ecuador. paulina.bolanos@espoch.edu.ec

${ }^{4}$ Escuela Superior Politécnica de Chimborazo, Facultad de Administración de Empresas. Riobamba, Ecuador. jennisa05@yahoo.es
} 
Palabras claves: neuroeconomía, neurociencia, comportamiento del consumidor, neuromarketing

\section{Introducción}

La neuroeconomía nace como una ciencia que pone en duda la teoría clásica del comportamiento del consumidor. Los mercados son cambiantes, las realidades fluctúan con el paso del tiempo, dando lugar a nuevos aportes de la neurociencia en la parte social y más aún en el comportamiento del ser humano que tienen incidencia directa en la actividad económica del mismo. Los mercados se abren a nuevos paradigmas del consumidor, temas que ya se consideraban desde hacía mucho tiempo, desde la creación de teorías económicas de Carlos Marx, se intuían ya las ideas de que el ser humano es un ser emocional, que requiere de bienes y servicios que le añadan emoción a su vida.

El estudio del cerebro y sus componentes es el tema que por varios años ha generado grandes desafíos, en muchas disciplinas que abordan este argumento, y las ciencias económicas no se han quedado atrás, pensar en que es posible tomar decisiones en política pública que consideren la parte humana y su beneficio, es la manera en la que el sistema económico sería totalmente compatible con las necesidades de sus habitantes. La ciencia económica reconoce que el consumidor toma decisiones influenciados por la parte afectiva y la parte deliberativa; y que es la parte deliberativa la que corrige los riesgos existentes en las decisiones emocionales, de esta manera se comprueba que la teoría clásica del comportamiento del consumidor está obsoleta y la influencia de la neurociencia les da un cambio relevante a las ciencias económicas.

\section{Aproximación teórica}

La neuroeconomía surge a partir de la economía conductual y la economía experimental, según Mullainathan y Thaler (2000), la Economía Conductual es una disciplina que combina la economía y la psicología, que investiga lo que ocurre en los mercados cuando los agentes sufren limitaciones y complicaciones propias de los seres humanos, así también Glimcher, Camerer, Fehr, \& Poldrack, (2009), establecen que el estudio de la economía conductual permite dar respuesta a la pregunta de cómo los individuos procesan la información y sus elecciones. La segunda aplica métodos de laboratorio para estudiar las interacciones de los seres humanos en los contextos sociales gobernados por reglas explícitas o implícitas. (Smith 2005). 
ISSN: 2602-8506

Vol. 3, $\mathrm{N}^{\circ} 4 .$, p.05-19, octubre - diciembre, 2019

Si bien es cierto nada permanece estático, todo cambia, es así como los descubrimientos, la ciencia y los avances tecnológicos han demostrado que somos seres en evolución, al igual que nuestras decisiones de compra, gustos, preferencias y la manera en que nos comportamos frente al mercado varían.

Es este siglo donde el estudio del cerebro se acentúa para determinar los comportamientos de las personas, ante determinados factores, varios científicos se han propuesto en dar respuesta; por qué las personas deciden un objeto por encima de otro en sus decisiones de compra y qué factores son los que influyen en tales decisiones.

En este contexto las decisiones de compra ya no resultan ser tan sensatas, el ser humano se deja llevar por sus impulsos y emociones en lugar de la razón. "Las elecciones que ese hacen influyen en los demás y de la misma manera esas influyen en ellos. Las personas son influenciadas por la publicidad y ciertos factores exógenos (Bermejo \& Izquierdo, 2013).

De acuerdo a varios estudiosos de la rama de la economía, determinaron que esta disciplina es una ciencia exacta, siendo explicada matemática y estadísticamente, pero estos supuestos dejaron de creerse cuando Adam Smith señaló que el ego es parte esencial del hombre, buscando satisfacer lo que desea. "En la teoría económica se acuñó el término "homo economicus" designando al ser humano que se comporta de manera lógica y racional, siendo capaz de comprender las reglas de la economía y actuar en consecuencia, sin embargo estas acotaciones parten de un error base, ya que lo seres humanos no actúan de forma estrictamente racional, coincidiendo este planteamiento con lo expuesto por Adam Smith tiempo atrás y con varios estudios actuales que respaldan esta afirmación (Bermejo \& Izquierdo, 2013). Esto conlleva a que las personas se dejan llevar por las emociones y como resultado el proceso de toma de decisiones es inconsciente.

Durante la última década del siglo XX, denominada década del cerebro, el estudio sobre las estructuras y su funcionamiento creció exponencialmente, dando como resultado nuevos descubrimientos sobre como el sistema nervioso evalúa los riesgos, las recompensas y las probabilidades de éxito, dando como origen a una nueva disciplina llamada neuroeconomía (Bermejo \& Izquierdo, 2013). La neurociencia, ocupa un papel sumamente importante en la explicación de nuevos descubrimientos y de cómo estos tienden a combinarse con otras disciplinas, como la neuropsicología, neuro ética, neuromarketing, neuro filosofía, entre otros. 
ISSN: 2602-8506

Vol. 3, N4., p.05-19, octubre - diciembre, 2019

En base a estas nuevas perspectivas, surge la neuroeconomía, siendo una nueva disciplina que se encarga de estudiar la toma de decisiones, además de tratar de aplicar los conocimientos sobre el funcionamiento del cerebro humano dentro de la economía, en cuanto a cómo las personas toman decisiones económicas financieras (Bermejo P. E., 2012).

La neuroeconomía, busca dar una explicación acerca de estos acontecimientos que suceden en las decisiones de compra de las personas. La neuroeconomía es una nueva disciplina científica que crea un puente entre la investigación neurocientífica y la teoría económica (Braidot, 2005, pág. 16).

Para (Cisnero Enríquez, 2012) supone que los seres humanos toman decisiones irracionales y sesgadas. La mayoría de las decisiones que toma el ser humano, están influenciadas por tres factores: estado psicológico de la persona que toma la decisión (no es lo mismo tomar una decisión en estado de estrés, que estar en un momento relajado); El ambiente que rodea a la persona (el medio en que se desenvuelva influye en su conducta; Las ideas percibidas sobre las situaciones a las que se enfrenta (códigos emocionales).

En la vida corriente, el comportamiento humano es influenciado a menudo por sentimientos como la inseguridad o la confianza, aunque David Hume citado por (Martinez \& Juanatey, 2013), identificaba que el ser humano tiene una complejidad que hace muy difícil anticipar su comportamiento, de allí que la neuroeconomía busca sustentar las teorías microeconómicas en detalles acerca del funcionamiento del cerebro, en especial en funciones tales como la forma en que se toman decisiones, pensamiento estratégico e intercambio. (Arciniegas, Guerrero, \& Moreno, 2011).

Durante la última década la neuroeconomía se ha consolidado como una neuro disciplina que se ha enfocado a entender las bases neurobiológicas que constituyen la esencia de los procesos neurales que involucran o que se ponen en marcha cada vez que se toman decisiones. (Diaz, 2014), así la neuroeconomía y la toma de decisiones son importantes en la teoría del consumidor y la determinación de la utilidad de los agentes económicos racionales.

La posibilidad de procesar múltiples alternativas que faciliten una dirección de acción óptima es el fin mismo de estos estudios, valorar cómo el comportamiento económico puede afectar a nuestro cerebro para que el mismo comprenda dicha acción, y cómo los descubrimientos neurocientíficos aportan a la ciencia, limitando, guiando, o no, a los modelos de economía. 
Vol. 3, N4., p.05-19, octubre - diciembre, 2019

En este mismo contexto el estudio de los procesos mentales que determinan la toma de decisiones resultan ser aspectos importantes para el estudio e investigación en la neuroeconomía, procesos que, por cierto, parecen ser bastante distintos de lo que suponemos y siempre se enmarcará en una elección individual referenciada siempre en el bienestar que se obtendrá a partir de ella (Nicola, 2007)

La neuroeconomía abre un nuevo camino en la investigación y estudio sobre los modelos aplicados a la economía tradicional y la reacción del cerebro ante diversas situaciones, con el fin de evaluar los diferentes comportamientos en la toma de decisiones. El ser humano antepone las emociones y el sentido de ventaja al momento de adquirir, intercambiar o necesitar algo; tomando en consideración la motivación, confianza, aprendizaje o miedo, ya que sus decisiones no son realmente lo que suponen.

Las emociones y su influencia en la decisión de compra

Al buscar establecer modelos de comportamientos económicos la neuroeconomía no solo considera los factores financieros, sino también a otros como son los factores sociales y emocionales al momento de tomar una decisión, en otros aspectos mide los perfiles individuales que están ligados a variables de riesgos y empatía, al establecer todo el análisis en un contexto la neuroeconomía podrá facilitar la formulación de nuevas políticas económicas que consideren el comportamiento real de las personas, el cual representará un enorme desarrollo y beneficio para la empresa.

Las emociones tienen gran incidencia a la hora de fijar y percibir los precios, varios estudios demuestran que el ser humano actúa de manera más emocional, pues al establecer un precio escogen el más económico sin embargo al momento de presentarles dicho producto con características de elegancia su decisión sobre el precio aumenta por el placer emocional de poseer algo, ya que consideran que puede ser un producto exclusivo o que simplemente tiene una marca reconocida.

Lo avances de la neuroeconomía son importantes al evidenciar que el cerebro humano está preparado a reaccionar de forma inmediata e irracional ante una información que puede ser negativa o falsa como son las publicidades engañosas que ante una información verdadera la cual recoge experiencias o investigaciones previas. Los avances tecnológicos ayudan a obtener información de forma individual el estudio del funcionamiento del cerebro de cada individuo 
para ser analizado, todo esto permitirá conocer el perfil adecuado para tomar decisiones económicas.

La neurociencia y la teoría económica llegan a un mismo lugar para discernir la forma en que las decisiones del consumidor se toman, para entender cómo se vende un producto o un servicio, de acuerdo el nivel de comportamiento del consumidor.

Identificar los factores que influyen en el comportamiento de consumo tributa a afinar los programas de mercadotecnia, a afinar las estrategias y tácticas relacionándolas a las características del segmento de clientes reales y clientes potenciales. Si se conociera algunos de estos factores resulta posible estimular los deseos latentes del consumidor y se alcanzaría los objetivos comerciales trazados.

\section{Metodología}

La investigación es de tipo exploratoria y descriptiva, parte de relaciones ampliamente estudiadas en la teoría para crear un marco conceptual que fundamente el estudio de las variables más importantes. El estudio es de tipo transversal, se empleó un enfoque mixto (cualitativocuantitativo). La técnica empleada es la encuesta, el alcance de la investigación es de tipo exploratorio y descriptivo

Para la población o universo, se consideró la PEA de la ciudad de Riobamba que registra un total de 52068 habitantes en el perímetro urbano.

La selección de la muestra se hizo a través de la técnica de muestreo aleatorio simple. Para el calculó se utilizó la fórmula propuesta por Mason, Lind y Marcal (2002):

Donde:

Tabla 1. Simbología para el cálculo del tamaño de la muestra

\begin{tabular}{lll}
\hline Símbolo & Significado & Valor \\
\hline $\mathrm{n}$ & Tamaño de la muestra & 382 \\
$\mathrm{~N}$ & El tamaño de la población & 52068 \\
$\mathrm{Z}$ & Valor obtenido mediante nivel de & 1,96 \\
$\mathrm{p}$ & confianza & \\
$\mathrm{q}$ & Probabilidad de ocurrencia de evento & 0,5 \\
$\mathrm{E}$ & Probabilidad de no ocurrencia de & 0,5 \\
\hline
\end{tabular}

Elaborado por: Los autores 
Aplicación de la formula

$$
n=\frac{N z^{2} p q}{E^{2}(N-1)+z^{2} p q} \quad n=\frac{50006,10}{131.13} \quad n=382
$$

La muestra mínima necesaria, tomando en cuenta estas consideraciones, es de 382

Se realizaron los respectivos análisis de confiabilidad y validez del cuestionario.

\section{Resultados}

P1 ¿Qué lugar prefiere para adquirir productos de primera necesidad?

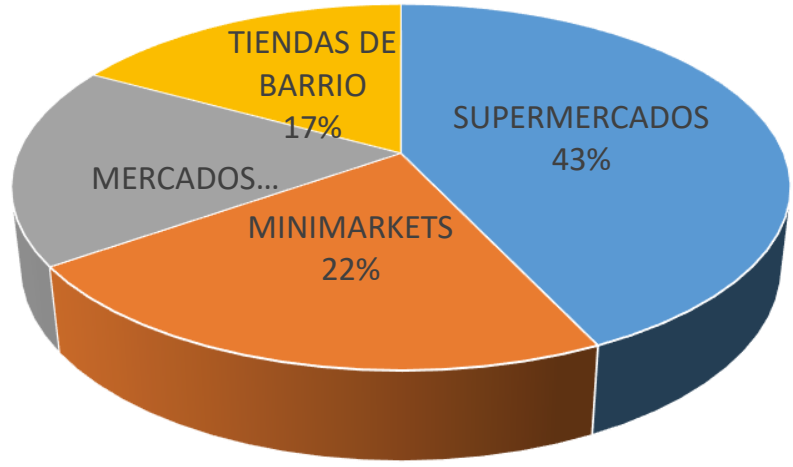

Figura 1. Lugar de preferencia para adquirir productos de primera necesidad Fuente: Elaboración propia

De los resultados se puede inferir que del 100\% de encuestados, el $43 \%$ prefiere adquirir productos de primera necesidad en supermercados, a diferencia del 17\% que prefiere visitar las tiendas de barrio. Los mercados populares y minimarkets son preferidos por un $18 \%$ y $22 \%$ de los encuestados.

P2. ¿Qué lugar prefiere para adquirir sus prendas de vestir?

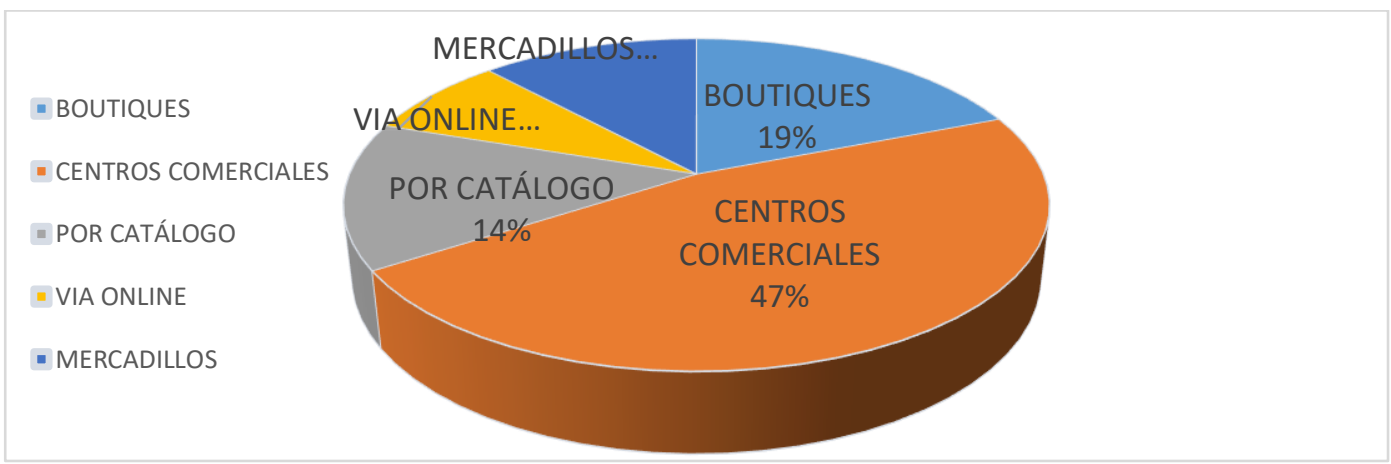

Figura 2. Lugar de preferencia para adquirir prendas de vestir Fuente: Elaboración propia 
Se evidencia que, del total de encuestados, el $47 \%$ prefiere comprar sus prendas de vestir en centros comerciales, seguido de un $19 \%$ que compra en boutiques, el 14\% prefiere las ventas por catálogo, un $12 \%$ adquiere sus prendas de vestir en mercadillos, y apenas un $8 \%$ las realiza a través de tiendas online.

P3. ¿La mayor parte de sus ingresos los destina a la compra de?

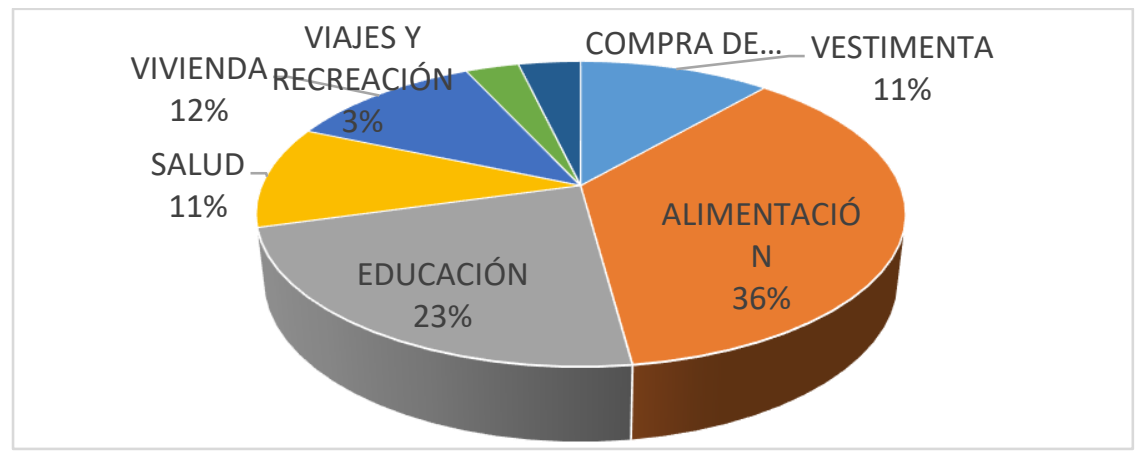

Figura 3. Destino de ingresos para comprar

Fuente: Elaboración propia

En el gráfico 3, se puede observar que el $36 \%$ de los encuestados destinan la mayor parte de sus ingresos para alimentación, el 23\% para educación, mientras que el $12 \%$ de personas invierte la mayor parte de sus ingresos en vivienda, en gastos de vestimenta se registra un porcentaje casi similar equivalente al $11 \%$, por otro lado se puede visualizar que la menor inversión se realiza en la adquisición de artículos tecnológicos y, en viajes y recreación con 4 y 3 por ciento de preferencia respectivamente.

P4. ¿Cuándo dispone de dinero usted prefiere invertirlo en?

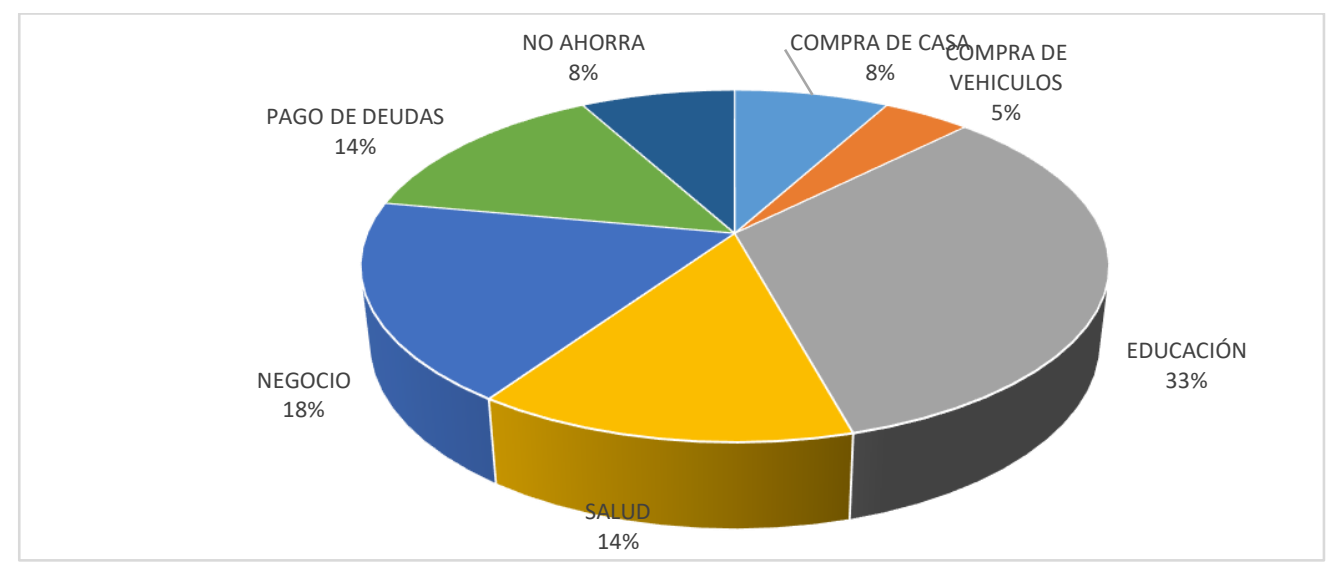

Figura 4. Destino de ingresos para invertir

Fuente: Elaboración propia 
ISSN: 2602-8506

Vol. 3, N4., p.05-19, octubre - diciembre, 2019

De los resultados se puede inferir que el $33 \%$ de las personas encuestadas prefieren invertir su dinero en educación, un 18\% lo destina a la adquisición o mejoras de su propio negocio, un 14\% lo destina al pago de deudas un porcentaje similar prefiere invertirlo en salud, un $8 \%$ a la adquisición de bienes inmuebles, en contraposición se registra que un $8 \%$ no ahorra, y apenas un 5\% decide invertirlo a la comprar de vehículos.

P5. ¿El factor determinante para decidir la compra de un producto es?:

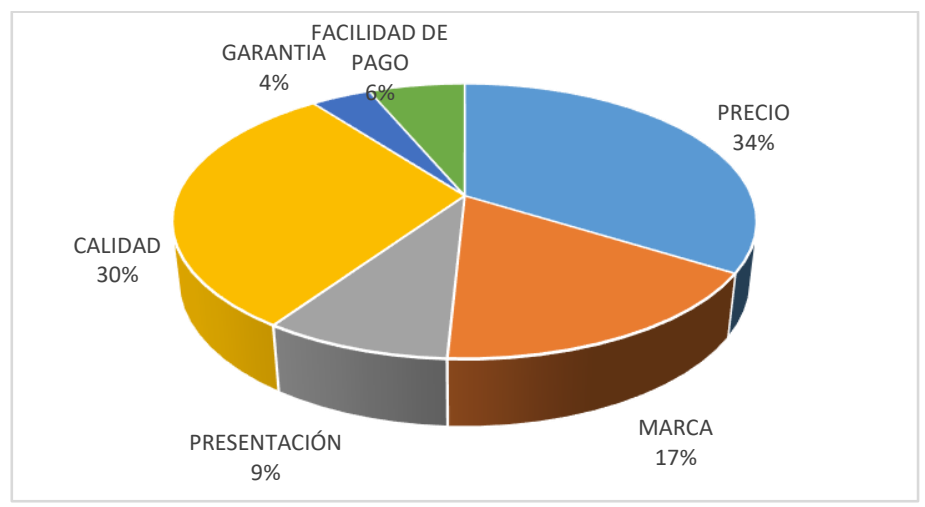

Figura 5. Factor determinante para elegir un producto

Fuente: Elaboración propia

En el gráfico 5, se puede observar que en un $34 \%$ de los sujetos de estudio el determinante de compra es el precio, seguido de un $30 \%$ cuyo factor determinante es la calidad, $17 \%$ considera que un determinante para realizar la compra de un producto es la marca, mientras que un $9 \%$ considera que la presentación del producto, un $6 \%$ considera las facilidades de pago y por último la garantía del producto es seleccionada apenas por un $4 \%$ de personas consultadas.

P6. ¿Con qué frecuencia solicita la recomendación de un amigo o familiar antes de realizar la comprar un producto?

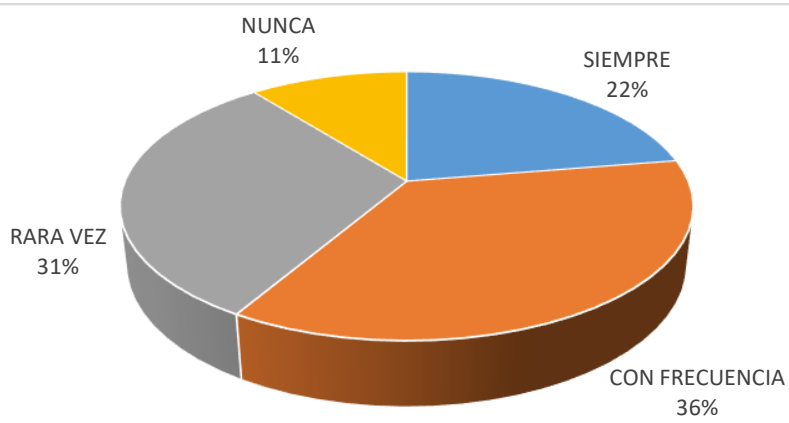

Figura 6. Recomendación de un amigo o familiar

Fuente: Elaboración propia 
ISSN: 2602-8506

Vol. 3, N4., p.05-19, octubre - diciembre, 2019

Como lo demuestra la gráfica que antecede, un $36 \%$ de los encuestados con frecuencia recurre a la recomendación de un amigo o familiar para comprar un producto, el $31 \%$ menciona que rara vez pide alguna recomendación, el 22\% manifiesta siempre pedir la recomendación, mientras que el $11 \%$ expone nunca pedir recomendación.

P7. ¿Qué tan importante es el servicio que recibe al momento de realizar una compra?

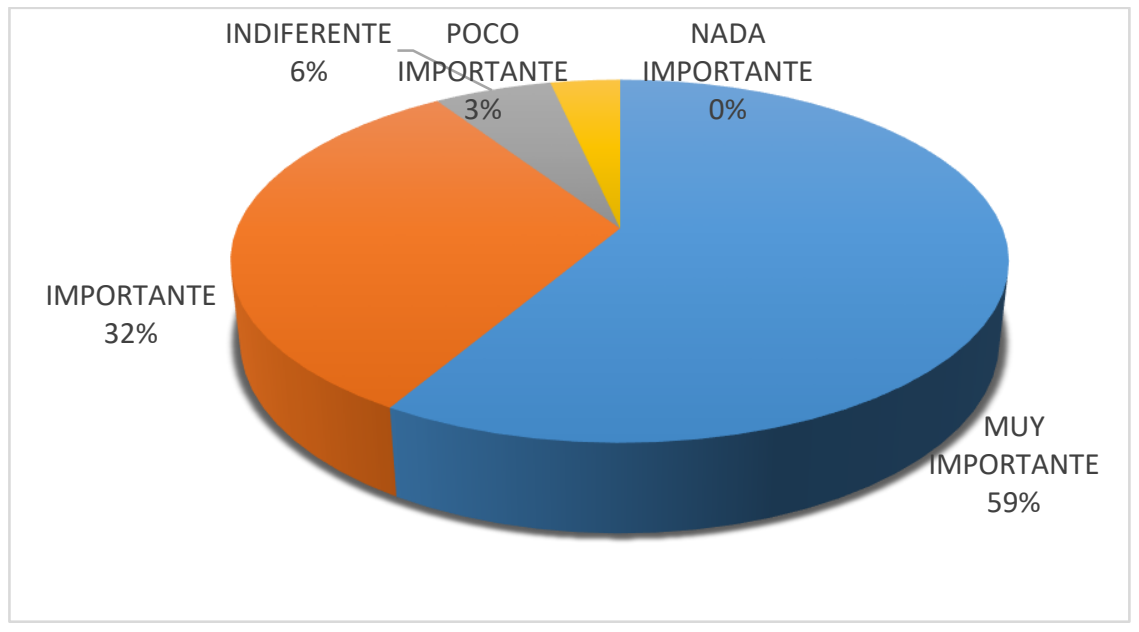

Figura 7. Importancia del servicio recibido

Fuente: Autores del estudio

En el gráfico 7, se puede ver que el 59\% de los encuestados considera muy importante el servicio que recibe al momento de realizar una compra, el $32 \%$ considera importante el servicio recibido, el $6 \%$ es indiferente respecto al servicio, mientras que el $3 \%$ considera poco importante.

P8. ¿Cuál considera usted, es la causa más importante por la que un cliente no regresa a comprar en un mismo establecimiento?

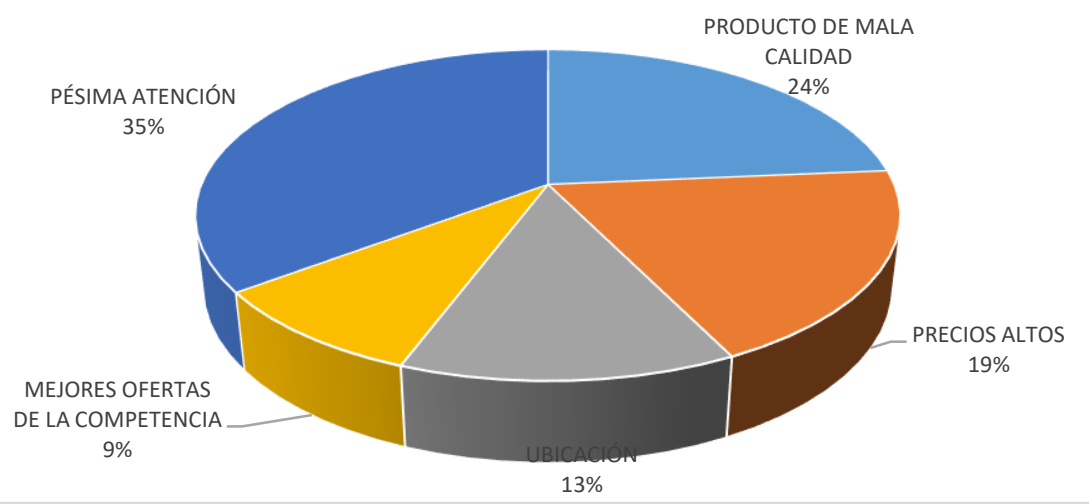

Figura 8. Causa importante por la que un cliente no regresa a comprar

Fuente: Elaboración propia 
ISSN: 2602-8506

Vol. 3, N4., p.05-19, octubre - diciembre, 2019

Según el $35 \%$ de encuetados el recibir una pésima atención es la causa más importante por la que no regresaría a comprar en un establecimiento, para el $24 \%$ la mala calidad de los productos, el 19\% considera una razón importante los precios altos, mientras que para el 13\% de encuestados la ubicación del establecimiento es determinante para no volver a realizar compras en el mismo lugar, y apenas un $9 \%$ considera que las mejores ofertas de la competencia como una posible razón para no volver a realizar compras en el mismo lugar.

P9. La mayor parte de las decisiones de compra en su hogar las realiza:

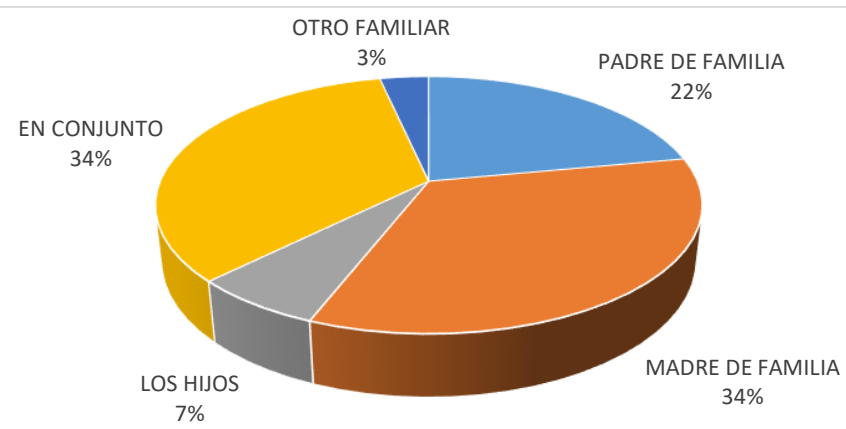

Figura 9. Miembro de la familia que toma la decisión de compra en el hogar

Fuente: Elaboración propia

La mayor parte en la decisión de compra de artículos para el hogar según el estudio realizado lo tiene la madre de familia según el 34\% de encuestados, igual porcentaje opina que la decisión se la toma en conjunto, mientras que el $22 \%$ indica que el padre de familia tiene la decisión de compra, el $7 \%$ opina que los hijos y apenas un 3\% indica que otro familiar tiene la decisión de compra de artículos para el hogar.

P10. ¿Qué tipo de institución prefiere para solicitar un servicio financiero?

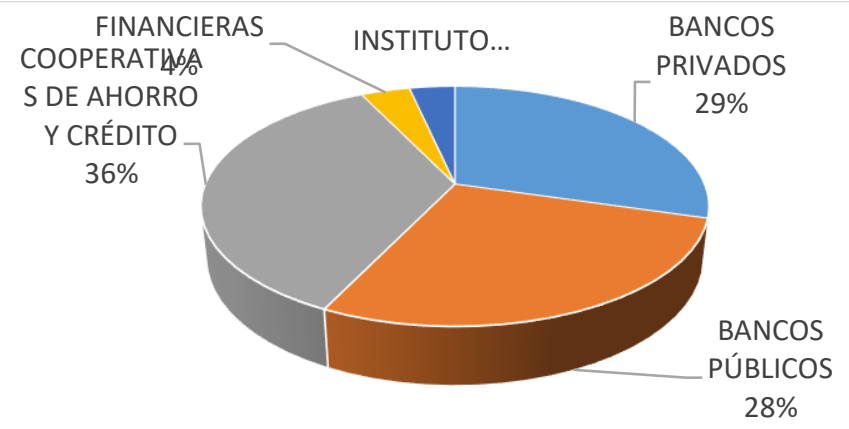

Figura 10. Tipo de institución preferida para solicitar servicios financieros

Fuente: Elaboración propia 
ISSN: 2602-8506

Vol. 3, N4., p.05-19, octubre - diciembre, 2019

De los datos que se obtuvieron se puedo inferir que el 36\% de los encuestados prefiere solicitar los servicios financieros en cooperativas de ahorro y crédito, el $29 \%$ prefiere hacerlo bancos privados a diferencia del $28 \%$ que selecciona bancos públicos, mientras que un $4 \%$ se inclina por utilizar otras financieras y apenas un 3\% opta por el IEES como prestador de servicios financieros.

P11. ¿Qué medios de comunicación utiliza para buscar información sobre los servicios que ofrece un establecimiento en particular?

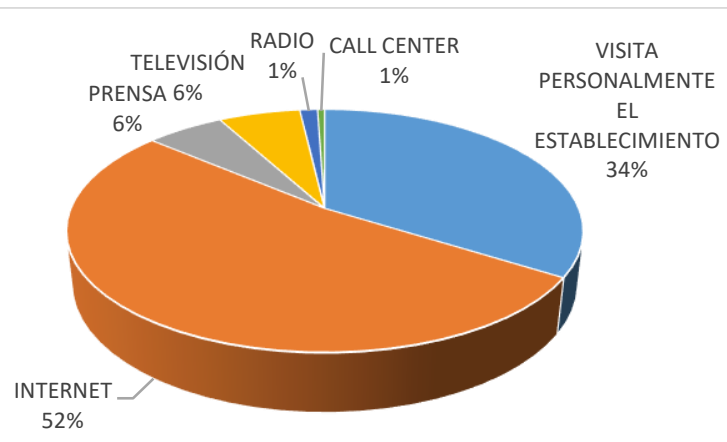

Figura 11. Medios utilizados para la búsqueda de información

Fuente: Elaboración propia

El 52\% de los encuestados optan por utilizar las diversas herramientas del internet para la búsqueda de información referente a un local comercial de interés, el 34\% prefiere visitar personalmente el establecimiento, la prensa y televisión coinciden con un $6 \%$ de interés respectivamente, mientras que la radio y los servicios de call center tienen preferencia del $1 \%$ respectivamente.

P12. ¿Es importante que los establecimientos comerciales cuenten con servicio de pago a través de tarjeta de crédito?

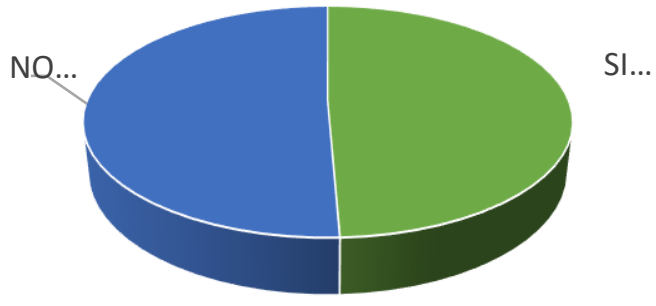

Figura 12. Importancia del uso de tarjetas de crédito para efectuar pagos

Fuente: Elaboración propia 


\section{Conclusiones}

Luego de procesar y analizar la información se arriba a las siguientes conclusiones

- El lugar de preferencia del consumidor riobambeño para la adquisición de productos de primera necesidad son los supermercados.

- Para la adquisición de prendas de vestir son los centros comerciales quienes ocupan el primer lugar.

- La mayor parte de los ingresos recibidos, los destina a la compra de alimentos, educación, y vestimenta en este orden de prioridad.

- El dinero producto de su ahorro es invertido en educación en un gran porcentaje.

- Los principales determinantes para la elección de un producto o servicio es el precio y la calidad.

- Las recomendaciones de un familiar o amigo son importantes al momento de decidir la compra.

- La calidad del servicio resulta ser un factor importante para la fidelización del cliente.

- La mala calidad del producto y la deficiente atención prestada constituyen detonantes para que el cliente no repita la compra.

- Las madres de familia dentro del núcleo familiar, son aquellas que deciden la compra o el uso de bienes y servicios en su gran mayoría.

- Las cooperativas de ahorro y crédito son las instituciones financieras de preferencia al momento de elegir un servicio financiero.

- Las redes sociales y las páginas web son los medios de comunicación más utilizados por el consumidor riobambeño al momento de buscar información de algún establecimiento en particular.

- Resulta de gran interés para los sujetos de estudio que los establecimientos comerciales cuenten con servicios de pagos a través de tarjetas de crédito.

- El estudio del comportamiento del consumidor es sin lugar a dudas el primer paso para la definición de una estrategia comercial, el éxito o el fracaso de un plan de mercadeo radica en la efectividad de la información recabada en el estudio del mercado.

\section{Referencias bibliográficas}

Arciniegas, A., Guerrero, J., \& Moreno, G. (2011). Una primera aproximación a la evaluación del impacto de la neuroeconomía para la teoría económica. Colombia.

Bermejo, P. E. (2012). Burbujas financieras y crisis económicas. Una aproximación desde la neurociencia. Kranion. Obtenido de http://www.kranion.es/images/2012_09_01_011016.pdf

Bermejo, P., \& Izquierdo, R. (2013). Tu dinero y tu cerebro: Por qué tomamos decisiones erróneas y cómo evitarlas. Madrid: Conecta. 
Vol. 3, No4., p.05-19, octubre - diciembre, 2019

Braidot, N. (2005). Neuromarketing: Neuroeconomía y Negocios. Buenos Aires: Puerto Norte

Sur. Obtenido de

https://books.google.com.ec/books?hl=es\&lr=\&id=yWiysczvwM8C\&oi=fnd\&pg=PA14\& $\mathrm{dq}=$ neuroeconom $\% \mathrm{C} 3 \% \mathrm{ADa} \& \mathrm{ots}=9 \mathrm{Kx}-$

UsSRSa\&sig=Lpxq0m9HgZC1o7A1TIVyhLdmqm8\#v=onepage \&q=neuroeconom\%C3\% $\mathrm{ADa} \& \mathrm{f}=\mathrm{false}$

Cisnero Enríquez, A. (2012). Neuromarketing y neuroeconomía: código emocional del consumidor. Bogotá: Ecoe Ediciones.

Diaz, H. (2014). Evolutionary Biology, brain and advertising: Neuroeconomics and neuromarketing in action (Vol. Comunicación y Cultura Vol. 2). Obtenido de https://www.realidadeconomica.umich.mx/index_files/bases_de_la_neuroeconomia_4.pdf

Glimcher, P., Camerer, C., Fehr, E., \& Poldrack, R. (2009). Introduction: A Brief History of Neuroeconomics. En P. Glimcher, C. Camerer, E. Fehr, \& R. Poldrack, Neuroeconomics (págs. 1-12). Londres: Elsevier

Martínez, V., \& Juanatey, O. (2013). Neuroeconomía y Neuromarketing.: Estado de la cuestión de las relaciones entre Neurociencias, Marketing y Economía. Coruña - España.

Mullainathan, S. \& Thaler, R. (2000) "Behavioural Economics", Working Paper 7948.

National Bureau of Economic Research.

Nicola, J. (2007). De la neurociencia a la neuroeconomía". Obtenido de http://defenderlapatria.com/130.13.pdf

Smith, V. (2005). ¿Qué es la economía experimental? Apuntes del Cenes [en línea] 2005, 25 (enero-junio) [Fecha de consulta: 10 de diciembre de 2018] Disponible en:〈http://www.redalyc.org/articulo.oa?id=479548746002> ISSN 0120-3053

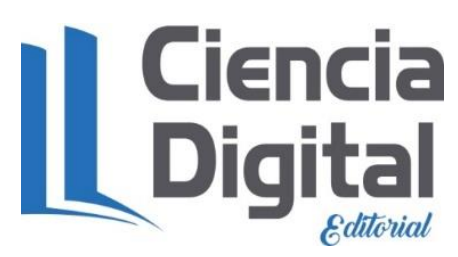


ISSN: 2602-8506

Vol. 3, N4., p.05-19, octubre - diciembre, 2019

\section{PARA CITAR EL ARTÍCULO INDEXADO}

Sánchez Lunavictoria, J., Delgado Rodriguez, C., Bolaños Logroño, P., \& Chávez Rojas, J. (2019). La neuroeconomía y su contribución a la teoría del comportamiento del consumidor. Visionario Digital, 3(4), 5-19. https://doi.org/10.33262/visionariodigital.v3i4.949

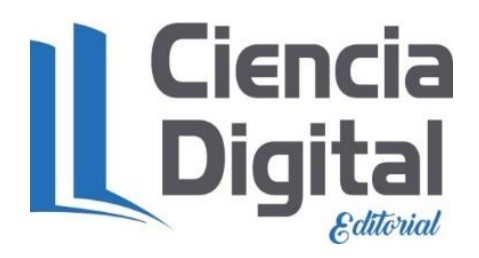

El artículo que se publica es de exclusiva responsabilidad de los autores y no necesariamente reflejan el pensamiento de la Revista Visionario Digital.

El artículo queda en propiedad de la revista y, por tanto, su publicación parcial y/o total en otro medio tiene que ser autorizado por el director de la Revista Visionario Digital.
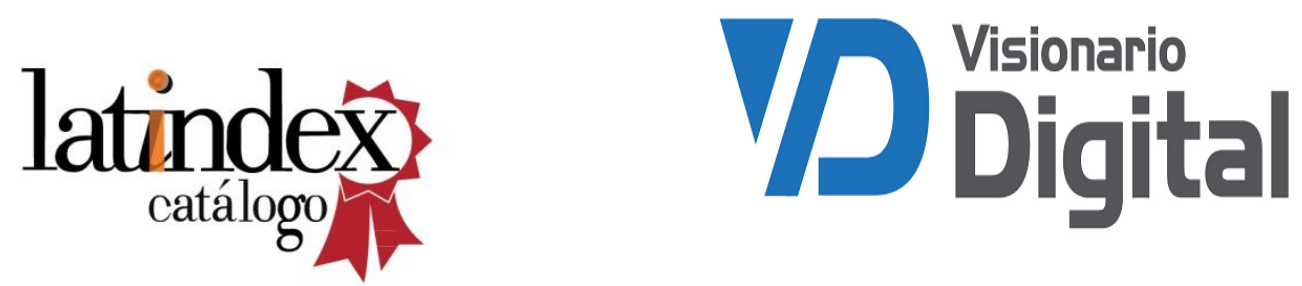